PROCEEDINGS OF THE

AMERICAN MATHEMATICAL SOCIETY

Volume 127, Number 4, April 1999, Pages 1163-1170

S 0002-9939(99)05050-9

\title{
FIXED POINT ITERATION FOR PSEUDOCONTRACTIVE MAPS
}

\author{
C. E. CHIDUME AND CHIKA MOORE
}

(Communicated by David R. Larson)

\begin{abstract}
Let $K$ be a compact convex subset of a real Hilbert space, $H ; T$ : $K \rightarrow K$ a continuous pseudocontractive map. Let $\left\{a_{n}\right\},\left\{b_{n}\right\},\left\{c_{n}\right\},\left\{a_{n}^{\prime}\right\},\left\{b_{n}^{\prime}\right\}$ and $\left\{c_{n}^{\prime}\right\}$ be real sequences in $[0,1]$ satisfying appropriate conditions. For arbitrary $x_{1} \in K$, define the sequence $\left\{x_{n}\right\}_{n=1}^{\infty}$ iteratively by $x_{n+1}=a_{n} x_{n}+$ $b_{n} T y_{n}+c_{n} u_{n} ; y_{n}=a_{n}^{\prime} x_{n}+b_{n}^{\prime} T x_{n}+c_{n}^{\prime} v_{n}, n \geq 1$, where $\left\{u_{n}\right\},\left\{v_{n}\right\}$ are arbitrary sequences in $K$. Then, $\left\{x_{n}\right\}_{n=1}^{\infty}$ converges strongly to a fixed point of $T$. A related result deals with the convergence of $\left\{x_{n}\right\}_{n=1}^{\infty}$ to a fixed point of $T$ when $T$ is Lipschitz and pseudocontractive. Our theorems also hold for the slightly more general class of continuous hemicontractive nonlinear maps.
\end{abstract}

\section{INTRODUCTION}

Let $H$ be a Hilbert space. A mapping $T: H \rightarrow H$ is said to be pseudocontractive (see e.g., [1], [2]) if

$$
\|T x-T y\|^{2} \leq\|x-y\|^{2}+\|(I-T) x-(I-T) y\|^{2}, \forall x, y \in H
$$

and is said to be strongly pseudocontractive if there exists $k \in(0,1)$ such that

$$
\|T x-T y\|^{2} \leq\|x-y\|^{2}+k\|(I-T) x-(I-T) y\|^{2}, \forall x, y \in H .
$$

Let $F(T):=\{x \in H: T x=x\}$ and let $K$ be a nonempty subset of $H$. A map $T: K \rightarrow K$ is called hemicontractive if $F(T) \neq \emptyset$ and

$$
\left\|T x-x^{*}\right\|^{2} \leq\left\|x-x^{*}\right\|^{2}+\|x-T x\|^{2} \forall x \in H, x^{*} \in F(T) .
$$

It is easy to see that the class of pseudocontractive maps with fixed points is a subclass of the class of hemicontractions. The following example, due to Rhoades [25], shows that the inclusion is proper. For $x \in[0,1]$, define $T:[0,1] \rightarrow[0,1]$ by $T x=\left(1-x^{\frac{2}{3}}\right)^{\frac{3}{2}}$. It is shown in [25] that $T$ is not Lipschitz and so cannot be nonexpansive. A straightforward computation (see e.g., [28]) shows that $T$ is pseudocontractive. For the importance of fixed points of pseudocontractions the reader may consult [1].

In the last ten years or so, numerous papers have been published on the iterative approximation of fixed points of Lipschitz strongly pseudocontractive (and correspondingly Lipschitz strongly accretive) maps using the Mann iteration process (see e.g., [17]). Results which had been known only in Hilbert spaces and only for Lipschitz maps have been extended to more general Banach spaces (see e.g., [3]-[6], [7], [8], [9], [10], [11], [12], [13], [16], [21]-[24], [25], [26]-[28], [29], [30], [31], [32] and the

Received by the editors August 1, 1997.

1991 Mathematics Subject Classification. Primary 47H05, 47H06, 47H10, 47H15.

(C)1999 American Mathematical Society 
references cited therein) and to more general classes of maps (see e.g., [4]-[6], [7], [8], [9], [10], [11], [12], [13], [14], [16], [18]-[20], [21]-[24], [26]-[28], [29], [30], [31], [32] and the references cited therein). This success, however, has not carried over to arbitrary Lipschitz pseudocontraction $T$ even when the domain of the operator $T$ is a a compact convex subset of a Hilbert space. In fact, it is still an open question whether or not the Mann iteration process converges under this setting. In 1974, Ishikawa introduced an iteration process which, in some sense, is more general than that of Mann and which converges, under this setting, to a fixed point of $T$. He proved the following theorem.

Theorem I (Ishikawa [15]). If $K$ is a compact convex subset of a Hilbert space $H$, $T: K \mapsto K$ is a Lipschitzian pseudocontractive map and $x_{0}$ is any point in $K$, then the sequence $\left\{x_{n}\right\}_{n \geq 0}$ converges strongly to a fixed point of $T$, where $x_{n}$ is defined iteratively for each positive integer $n \geq 0$ by

$$
x_{n+1}=\left(1-\alpha_{n}\right) x_{n}+\alpha_{n} T\left[\left(1-\beta_{n}\right) x_{n}+\beta_{n} T x_{n}\right]
$$

where $\left\{\alpha_{n}\right\},\left\{\beta_{n}\right\}$ are sequences of positive numbers satisfying the conditions

$$
\text { (i) } 0 \leq \alpha_{n} \leq \beta_{n}<1 ; \text { (ii) } \lim _{n \rightarrow \infty} \beta_{n}=0 ; \text { (iii) } \sum_{n \geq 0} \alpha_{n} \beta_{n}=\infty .
$$

Since its publication in 1974, Theorem I, as far as we know, has never been extended to more general Banach spaces. In [18], Qihou extended the theorem to the slightly more general class of Lipschitz hemicontractions and in [19] he proved, under the setting of Theorem I, that the convergence of the recursion formula (3) to a fixed point of $T$ when $T$ is a continuous hemicontractive map, under the additional hypothesis that the number of fixed points of $T$ is finite. The iteration process (3) is generally referred to as the Ishikawa iteration process in light of [15]. Another iteration process which has been studied extensively in connection with fixed points of pseudocontractive maps is the following: For $K$ a convex subset of a Banach space $E$, and $T: K \rightarrow K$, the sequence $\left\{x_{n}\right\}_{n=1}^{\infty}$ is defined iteratively by $x_{1} \in K$,

$$
x_{n+1}=\left(1-c_{n}\right) x_{n}+c_{n} T x_{n}, n \geq 1,
$$

where $\left\{c_{n}\right\}$ is a real sequence satisfying the following conditions: $(i) 0 \leq c_{n}<$ 1 ; (ii) $\lim c_{n}=0$; (iii) $\sum_{n=1}^{\infty} c_{n}=\infty$. The iteration process (4) is generally referred to as the Mann iteration process in light of [17].

In 1995, Liu [16] introduced what he called Ishikawa and Mann iteration processes with errors as follows:

(a) For $K$ a nonempty subset of $E$ and $T: K \rightarrow E$, the sequence $\left\{x_{n}\right\}$ defined by $x_{1} \in K, x_{n+1}=\left(1-\alpha_{n}\right) x_{n}+\alpha_{n} T y_{n}+u_{n} ; y_{n}=\left(1-\beta_{n}\right) x_{n}+\beta_{n} T x_{n}+v_{n}, n \geq$ 1 where, $\left\{\alpha_{n}\right\},\left\{\beta_{n}\right\}$ are sequences in $[0,1]$ satisfying appropriate conditions and $\sum\left\|u_{n}\right\|<\infty, \sum\left\|v_{n}\right\|<\infty$ is called the Ishikawa Iteration process with errors.

(b) With $K, E$ and $T$ as in part (a), the sequence $\left\{x_{n}\right\}$ defined by $x_{1} \in K$, $x_{n+1}=\left(1-\alpha_{n}\right) x_{n}+\alpha_{n} T x_{n}+u_{n}, n \geq 1$ where $\left\{\alpha_{n}\right\}$ is a sequence in $[0,1]$ satisfying appropriate conditions and $\sum\left\|u_{n}\right\|<\infty$, is called the Mann iteration process with errors.

While it is known that consideration of error terms in iterative processes is an important part of the theory, it is also clear that the iteration processes with errors introduced by Liu in (a) and (b) are unsatisfactory. The occurrence of errors is random so that the conditions imposed on the error terms in (a) and (b) which 
imply, in particular, that they tend to zero as $n$ tends to infinity are, therefore, unreasonable. Recently, Yuguang Xu [30] introduced the following more satisfactory definitions.

[A] Let $K$ be a nonempty convex subset of $E$ and $T: K \rightarrow K$ a mapping. For any given $x_{1} \in K$, the sequence $\left\{x_{n}\right\}_{n=1}^{\infty}$ defined iteratively by

$$
x_{n+1}=a_{n} x_{n}+b_{n} T y_{n}+c_{n} u_{n} ; y_{n}=a_{n}^{\prime} x_{n}+b_{n}^{\prime} T x_{n}+c_{n}^{\prime} v_{n}, n \geq 1
$$

where $\left\{u_{n}\right\},\left\{v_{n}\right\}$ are bounded sequences in $K$ and $\left\{a_{n}\right\},\left\{b_{n}\right\},\left\{c_{n}\right\},\left\{a_{n}^{\prime}\right\},\left\{b_{n}^{\prime}\right\}$ and $\left\{c_{n}^{\prime}\right\}$ are sequences in $[0,1]$ such that $a_{n}+b_{n}+c_{n}=a_{n}^{\prime}+b_{n}^{\prime}+c_{n}^{\prime}=1 \forall n \geq 1$ is called the Ishikawa iteration sequence with errors.

[B] If, with the same notations and definitions as in [A], $b_{n}^{\prime}=c_{n}^{\prime}=0$, for all integers $n \geq 1$, then the sequence $\left\{x_{n}\right\}_{n=1}^{\infty}$ now defined by $x_{1} \in K, x_{n+1}=$ $a_{n} x_{n}+b_{n} T x_{n}+c_{n} u_{n}, n \geq 1$, is called the Mann iteration sequence with errors. We remark that if $K$ is bounded (as is generally the case), the error terms $u_{n}, v_{n}$ are arbitrary in $K$.

It is our purpose in this paper to extend Theorem I to the Ishikawa iteration process with errors in the sense of $[\mathrm{A}]$ and to the slightly more general class of Lipschitz hemicontractions. Our theorem will include Theorem 2 of Qihou [18] as a special case. Furthermore, we shall prove a theorem similar to Theorem I for continuous hemicontractions.

\section{Preliminaries}

We shall make use of the following results.

Lemma 1 ([29]). Suppose that $\left\{\rho_{n}\right\},\left\{\sigma_{n}\right\}$ are two sequences of nonnegative numbers such that for some real number $N_{0} \geq 1, \rho_{n+1} \leq \rho_{n}+\sigma_{n} \forall n \geq N_{0}$.

(a) If $\sum \sigma_{n}<\infty$, then, $\lim \rho_{n}$ exists.

(b) If $\sum \sigma_{n}<\infty$ and $\left\{\rho_{n}\right\}$ has a subsequence converging to zero, then $\lim \rho_{n}=0$.

We shall also use the following well-known identity for Hilbert spaces, $H$ :

$$
\begin{aligned}
\|(1-\lambda) x+\lambda y\|^{2}= & (1-\lambda)\|x\|^{2}+\lambda\|y\|^{2} \\
& -\lambda(1-\lambda)\|x-y\|^{2} \forall x, y \in H, \lambda \in[0,1] .
\end{aligned}
$$

\section{MAIN THEOREMS}

We prove the following theorems.

Theorem 1. Let $K$ be a compact convex subset of a real Hilbert space, $H$; $T$ : $K \rightarrow K$ a continuous hemicontractive map. Let $\left\{a_{n}\right\},\left\{b_{n}\right\},\left\{c_{n}\right\},\left\{a_{n}^{\prime}\right\},\left\{b_{n}^{\prime}\right\}$ and $\left\{c_{n}^{\prime}\right\}$ be real sequences in $[0,1]$ satisfying the following conditions:

(i) $a_{n}+b_{n}+c_{n}=a_{n}^{\prime}+b_{n}^{\prime}+c_{n}^{\prime}=1 \forall n \geq 1$;

(ii) $\lim b_{n}=\lim b_{n}^{\prime}=0$;

(iii) $\sum c_{n}<\infty ; \sum c_{n}^{\prime}<\infty$;

(iv) $\sum \alpha_{n} \beta_{n}=\infty ; \sum \alpha_{n} \beta_{n} \delta_{n}<\infty$, where $\delta_{n}:=\left\|T x_{n}-T y_{n}\right\|^{2}$;

(v) $0 \leq \alpha_{n} \leq \beta_{n}<1 \forall n \geq 1$, where $\alpha_{n}:=b_{n}+c_{n} ; \beta_{n}:=b_{n}^{\prime}+c_{n}^{\prime}$.

For arbitrary $x_{1} \in K$, define the sequence $\left\{x_{n}\right\}_{n=1}^{\infty}$ iteratively by

$$
x_{n+1}=a_{n} x_{n}+b_{n} T y_{n}+c_{n} u_{n} ; y_{n}=a_{n}^{\prime} x_{n}+b_{n}^{\prime} T x_{n}+c_{n}^{\prime} v_{n}, n \geq 1,
$$


where $\left\{u_{n}\right\},\left\{v_{n}\right\}$ are arbitrary sequences in $K$. Then, $\left\{x_{n}\right\}_{n=1}^{\infty}$ converges strongly to a fixed point of $T$.

Proof. The existence of a fixed point of $T$ follows from Schauder's fixed point theorem. So $F(T) \neq \emptyset$. Let $x^{*} \in K$ be a fixed point of $T$. Using the identity (5), we obtain the following estimates: For some constants $M_{1} \geq 0, M_{2} \geq 0$,

$$
\begin{aligned}
\left\|y_{n}-x^{*}\right\|^{2}= & \left\|\left(1-\beta_{n}\right)\left(x_{n}-x^{*}\right)+\beta_{n}\left(T x_{n}-x^{*}\right)-c_{n}^{\prime}\left(T x_{n}-v_{n}\right)\right\|^{2} \\
\leq & \left(\left\|\left(1-\beta_{n}\right)\left(x_{n}-x^{*}\right)+\beta_{n}\left(T x_{n}-x^{*}\right)\right\|+c_{n}^{\prime}\left\|T x_{n}-v_{n}\right\|\right)^{2} \\
\leq & \left(1-\beta_{n}\right)\left\|x_{n}-x^{*}\right\|^{2}+\beta_{n}\left\|T x_{n}-x^{*}\right\|^{2} \\
& -\beta_{n}\left(1-\beta_{n}\right)\left\|x_{n}-T x_{n}\right\|^{2}+c_{n}^{\prime} M_{1} \\
\left\|y_{n}-T y_{n}\right\|^{2}= & \left\|\left(1-\beta_{n}\right)\left(x_{n}-T y_{n}\right)+\beta_{n}\left(T x_{n}-T y_{n}\right)-c_{n}^{\prime}\left(T x_{n}-v_{n}\right)\right\|^{2} \\
\leq & \left(1-\beta_{n}\right)\left\|x_{n}-T y_{n}\right\|^{2}+\beta_{n}\left\|T x_{n}-T y_{n}\right\|^{2} \\
& -\beta_{n}\left(1-\beta_{n}\right)\left\|x_{n}-T x_{n}\right\|^{2}+c_{n}^{\prime} M_{2}
\end{aligned}
$$

so using the fact that $T$ is hemicontractive with the estimates above, we obtain,

$$
\begin{aligned}
\left\|T x_{n}-x^{*}\right\|^{2} \leq & \left\|x_{n}-x^{*}\right\|^{2}+\left\|x_{n}-T x_{n}\right\|^{2} \\
\left\|T y_{n}-x^{*}\right\|^{2} \leq & \left\|y_{n}-x^{*}\right\|^{2}+\left\|y_{n}-T y_{n}\right\|^{2} \\
\leq & \left(1-\beta_{n}\right)\left\|x_{n}-x^{*}\right\|^{2}+\beta_{n}\left\|T x_{n}-x^{*}\right\|^{2} \\
& -\beta_{n}\left(1-\beta_{n}\right)\left\|x_{n}-T x_{n}\right\|^{2}+\left(1-\beta_{n}\right)\left\|x_{n}-T y_{n}\right\|^{2} \\
& +\beta_{n}\left\|T x_{n}-T y_{n}\right\|^{2}-\beta_{n}\left(1-\beta_{n}\right)\left\|x_{n}-T x_{n}\right\|^{2}+M_{3} c_{n}^{\prime} \\
\leq & \left\|x_{n}-x^{*}\right\|^{2}-\beta_{n}\left(1-2 \beta_{n}\right)\left\|x_{n}-T x_{n}\right\|^{2} \\
& +\left(1-\beta_{n}\right)\left\|x_{n}-T y_{n}\right\|^{2}+\beta_{n}\left\|T x_{n}-T y_{n}\right\|^{2}+M_{3} c_{n}^{\prime}
\end{aligned}
$$

where $M_{3}=M_{1}+M_{2}$. Therefore, for some constant $M_{4} \geq 0$,

$$
\begin{aligned}
\left\|x_{n+1}-x^{*}\right\|^{2} \leq & \left(1-\alpha_{n}\right)\left\|x_{n}-x^{*}\right\|^{2}+\alpha_{n}\left\|T y_{n}-x^{*}\right\|^{2} \\
& -\alpha_{n}\left(1-\alpha_{n}\right)\left\|x_{n}-T y_{n}\right\|^{2}+c_{n} M_{4} \\
\leq & \left(1-\alpha_{n}\right)\left\|x_{n}-x^{*}\right\|^{2}+\alpha_{n}\left[\left\|x_{n}-x^{*}\right\|^{2}\right. \\
& -\beta_{n}\left(1-2 \beta_{n}\right)\left\|x_{n}-T x_{n}\right\|^{2}+\left(1-\beta_{n}\right)\left\|x_{n}-T y_{n}\right\|^{2} \\
& \left.\quad+\beta_{n}\left\|T x_{n}-T y_{n}\right\|^{2}+M_{3} c_{n}^{\prime}\right] \\
& -\alpha_{n}\left(1-\alpha_{n}\right)\left\|x_{n}-T y_{n}\right\|^{2}+c_{n} M_{4} \\
= & \left\|x_{n}-x^{*}\right\|^{2}-\alpha_{n} \beta_{n}\left(1-2 \beta_{n}\right)\left\|x_{n}-T x_{n}\right\|^{2} \\
& +\alpha_{n} \beta_{n}\left\|T x_{n}-T y_{n}\right\|^{2}-\alpha_{n}\left(\beta_{n}-\alpha_{n}\right)\left\|x_{n}-T y_{n}\right\|^{2} \\
& +M_{5}\left(c_{n}+c_{n}^{\prime}\right)
\end{aligned}
$$

where $M_{5}=\max \left\{M_{3}, M_{4}\right\}$, since $\alpha_{n} \leq 1$. Thus, since $\beta_{n} \geq \alpha_{n}$, we have

$$
\begin{aligned}
\left\|x_{n+1}-x^{*}\right\|^{2} \leq & \left\|x_{n}-x^{*}\right\|^{2}-\alpha_{n} \beta_{n}\left(1-2 \beta_{n}\right)\left\|x_{n}-T x_{n}\right\|^{2} \\
& +\alpha_{n} \beta_{n}\left\|T x_{n}-T y_{n}\right\|^{2}+M_{5}\left(c_{n}+c_{n}^{\prime}\right) .
\end{aligned}
$$

Since $K$ is compact and $T$ is continuous, $\left\{\left\|x_{n}-T x_{n}\right\|\right\}$ is a bounded sequence. Let $\liminf _{n \rightarrow \infty}\left\|x_{n}-T x_{n}\right\|=\delta \geq 0$.

Claim. $\delta=0$.

Suppose the claim is false, that is, $\delta>0$. Then, there exists an integer $N_{1}>0$ such that $\left\|x_{n}-T x_{n}\right\| \geq \frac{\delta}{2} \forall n \geq N_{1}$. Observe that $\left\|x_{n}-y_{n}\right\|=\beta_{n}\left\|x_{n}-T x_{n}\right\|+$ 
$c_{n}^{\prime}\left\|T x_{n}-v_{n}\right\| \leq \operatorname{diam}(K)\left(\beta_{n}+c_{n}^{\prime}\right) \rightarrow 0$ as $n \rightarrow \infty$. Compactness of $K$ and continuity of $T$ imply that $\left\|T x_{n}-T y_{n}\right\| \rightarrow 0$ as $n \rightarrow \infty$. Thus, there exists an integer $N_{2}>0$ such that $\left\|T x_{n}-T y_{n}\right\| \leq \frac{\delta}{6} \forall n \geq N_{2}$. By conditions (ii) and (iii), there exists an integer $N_{3}>0$ such that $\beta_{n} \leq \frac{1}{3} \forall n \geq N_{3}$. Let $N=\max \left\{N_{1}, N_{2}, N_{3}\right\}$. Then, $\forall n \geq N$, inequality (8) now yields

$$
\begin{aligned}
\left\|x_{n+1}-x^{*}\right\|^{2} & \leq\left\|x_{n}-x^{*}\right\|^{2}-\alpha_{n} \beta_{n}\left(1-2 \beta_{n}\right) \frac{\delta^{2}}{4}+\alpha_{n} \beta_{n} \frac{\delta^{2}}{36}+M_{5}\left(c_{n}+c_{n}^{\prime}\right) \\
& \leq\left\|x_{n}-x^{*}\right\|^{2}-\frac{\delta^{2}}{18} \alpha_{n} \beta_{n}+M_{5}\left(c_{n}+c_{n}^{\prime}\right) ; \\
\Rightarrow \lambda \alpha_{n} \beta_{n} \leq\left\|x_{n}-x^{*}\right\|^{2}-\left\|x_{n+1}-x^{*}\right\|^{2}+M_{5}\left(c_{n}+c_{n}^{\prime}\right) ; \lambda=\frac{\delta^{2}}{18} & \Rightarrow \lambda \sum_{j=N}^{n} \alpha_{j} \beta_{j} \leq\left\|x_{N}-x^{*}\right\|^{2}-\left\|x_{n+1}-x^{*}\right\|^{2}+M \sum_{j=N}^{n}\left(c_{j}+c_{j}^{\prime}\right) .
\end{aligned}
$$

Since the right hand side is finite, this implies that $\sum_{j \geq N} \alpha_{j} \beta_{j}<\infty$ which contradicts hypothesis $(i v)$. Hence, $\liminf _{n \rightarrow \infty}\left\|x_{n}-T x_{n}\right\|=0$ and the claim is established. By compactness of $K$ this immediately implies that there is a subsequence $\left\{x_{n_{j}}\right\}$ of $\left\{x_{n}\right\}$ which converges to a fixed point of $T$, say $x^{*}$.

(For the rest of the argument, $x^{*}$ refers to this fixed point.) Let

$$
\Psi_{n}=\left\|x_{n}-x^{*}\right\|^{2} ; \quad \sigma_{n}=\alpha_{n} \beta_{n}\left\|T x_{n}-T y_{n}\right\|^{2}+M_{5}\left(c_{n}+c_{n}^{\prime}\right)
$$

and observe that $\Psi_{n} \geq 0, \sigma_{n} \geq 0, \forall n \geq 1$ and $\sum_{n \geq 1} \sigma_{n}<\infty$ by conditions (iii) and $(i v)$. Then, inequality (8) yields

$$
\Psi_{n+1} \leq \Psi_{n}+\sigma_{n}, \quad \forall n \geq 0
$$

It now follows from Lemma 1 that $\Psi_{n} \rightarrow 0$ as $n \rightarrow \infty$, i.e., $x_{n} \rightarrow x^{*}$ as $n \rightarrow \infty$. The proof is complete.

Corollary 1. Let $K$ be a compact convex subset of a real Hilbert space $H, T$ : $K \rightarrow K$ a Lipschitz hemicontractive map. Let $\left\{a_{n}\right\},\left\{b_{n}\right\},\left\{c_{n}\right\},\left\{a_{n}^{\prime}\right\},\left\{b_{n}^{\prime}\right\}$ and $\left\{c_{n}^{\prime}\right\}$ satisfy conditions $(i)$-(iii) of Theorem 1. Let $(i v) \sum \alpha_{n} \beta_{n}=\infty,(v) 0 \leq$ $\alpha_{n} \leq \beta_{n}<1 \forall n \geq 1$, where $\alpha_{n}, \beta_{n}$ are as defined in Theorem 1. For arbitrary $x_{1} \in K$, define the sequence $\left\{x_{n}\right\}_{n=1}^{\infty}$ iteratively by

$$
x_{n+1}=a_{n} x_{n}+b_{n} T y_{n}+c_{n} u_{n} ; y_{n}=a_{n}^{\prime} x_{n}+b_{n}^{\prime} T x_{n}+c_{n}^{\prime} v_{n}
$$

where $\left\{u_{n}\right\},\left\{v_{n}\right\}$ are arbitrary sequences in $K$. Then, $\left\{x_{n}\right\}_{n=1}^{\infty}$ converges strongly to a fixed point of $T$.

Proof. Since $T$ is hemicontractive, $F(T) \neq \emptyset$. Let $x^{*} \in F(T)$. As in the proof of Theorem 1, we obtain inequality (8). Let $L>0$ denote the Lipschitz constant of T. Then,

$$
\left\|T x_{n}-T y_{n}\right\| \leq L\left\|y_{n}-x_{n}\right\| \leq L\left\{\beta_{n}\left\|x_{n}-T x_{n}\right\|+c_{n}^{\prime} M_{6}\right\}
$$

for some constant $M_{6} \geq 0$. Thus, for some constant $M_{7} \geq 0$,

$$
\left\|T x_{n}-T y_{n}\right\|^{2} \leq L^{2} \beta_{n}^{2}\left\|x_{n}-T x_{n}\right\|^{2}+c_{n}^{\prime} M_{7}
$$

and so inequality (8) now yields, for some constant $M_{8} \geq 0$,

$$
\left\|x_{n+1}-x^{*}\right\|^{2} \leq\left\|x_{n}-x^{*}\right\|^{2}-\alpha_{n} \beta_{n}\left(1-2 \beta_{n}-L^{2} \beta_{n}^{2}\right)\left\|x_{n}-T x_{n}\right\|^{2}+c_{n}^{\prime} M_{8} .
$$


Since $\lim \beta_{n}=0$, there exists an integer $N_{8} \geq 1$ such that $2 \beta_{n}+L^{2} \beta_{n}^{2} \leq \frac{1}{2}$ so that

$$
\left\|x_{n+1}-x^{*}\right\|^{2} \leq\left\|x_{n}-x^{*}\right\|^{2}-\frac{1}{2} \alpha_{n} \beta_{n}\left\|x_{n}-T x_{n}\right\|^{2}+c_{n}^{\prime} M_{8} .
$$

The rest of the argument now follows as in the proof of Theorem 1 to yield that $x_{n} \rightarrow x^{*}$ as $n \rightarrow \infty$. The proof is complete.

Remark 1. Corollary 1 is an extension of Theorem I to the more general Ishikawa iteration sequence with errors in terms of [A] and to the slightly more general class of nonlinear Lipschitz hemicontractions. The corollary also extends Theorem 2 of Qihou [18] to the more general iterative scheme with errors.

Corollary 2. Let $K, H,\left\{a_{n}\right\},\left\{b_{n}\right\},\left\{c_{n}\right\},\left\{a_{n}^{\prime}\right\},\left\{b_{n}^{\prime}\right\},\left\{c_{n}^{\prime}\right\}, \alpha_{n}, \beta_{n} \quad$ be $\quad$ as in Theorem 1. Let $T: K \rightarrow H$ be a continuous hemicontractive map. Let $P_{K}: H \rightarrow K$ be the projection operator of $H$ onto $K$. Then, the sequence $\left\{x_{n}\right\}_{n=1}^{\infty}$ defined iteratively by

$$
\begin{gathered}
x_{n+1}=P_{K} z_{n} ; z_{n}=a_{n} x_{n}+b_{n} T y_{n}+c_{n} u_{n} ; \\
y_{n}=P_{K} \omega_{n} ; \omega_{n}=a_{n}^{\prime} x_{n}+b_{n}^{\prime} T x_{n}+c_{n}^{\prime} v_{n}, n \geq 1
\end{gathered}
$$

where $\left\{u_{n}\right\},\left\{v_{n}\right\}$ are arbitrary sequences in $K$, converges strongly to a fixed point of $T$.

Proof. The operator $P_{K}$ is nonexpansive (see e.g., [2]). $K$ is a Chebyshev subset of $H$ so that, $P_{K}$ is a single-valued map. Hence, we have the following estimates: For some constants $M_{9} \geq 0, M_{10} \geq 0$,

$$
\begin{aligned}
\left\|y_{n}-x^{*}\right\|^{2}= & \left\|P_{K} w_{n}-P_{K} x^{*}\right\|^{2} \leq\left\|w_{n}-x^{*}\right\|^{2} \\
= & \left(1-\beta_{n}\right)\left\|x_{n}-x^{*}\right\|^{2}+\beta_{n}\left\|T x_{n}-x^{*}\right\|^{2} \\
& \quad-\beta_{n}\left(1-\beta_{n}\right)\left\|x_{n}-T x_{n}\right\|^{2}+c_{n}^{\prime} M_{9}, \\
\left\|x_{n+1}-x^{*}\right\|^{2}= & \left\|P_{K} z_{n}-P_{K} x^{*}\right\|^{2} \\
\leq & \left\|z_{n}-x^{*}\right\|^{2} \\
= & \left\|x_{n}-x^{*}\right\|^{2}-\alpha_{n} \beta_{n}\left(1-2 \beta_{n}\right)\left\|x_{n}-T x_{n}\right\|^{2} \\
& +\alpha_{n} \beta_{n}\left\|T x_{n}-T y_{n}\right\|^{2}+M_{10}\left(c_{n}+c_{n}^{\prime}\right) .
\end{aligned}
$$

The set $K \cup T(K)$ is compact and so the sequence $\left\{\left\|x_{n}-T x_{n}\right\|\right\}$ is bounded. The rest of the argument follows exactly as in the proof of Theorem 1 and the proof is complete.

Remarks. 1. Conditions similar to our condition ( $i v)$ have been imposed in the literature. Reich [22] imposed the condition $\sum_{n=0}^{\infty} c_{n}^{2}\left\|T x_{n}\right\|^{2}<\infty$ (where $c_{n}$ is a real sequence in $(0,1)$ satisfying appropriate conditions) to prove the convergence of the Mann iteration process to the solution of an operator equation involving strongly accretive operator $T$ defined on a uniformly smooth Banach space.

2. A prototype for our parameters is

$$
\begin{gathered}
a_{n}=1-\frac{1}{\sqrt{(n+1)}}=a_{n}^{\prime} ; b_{n}=\frac{1}{\sqrt{(n+1)}}-\frac{1}{(n+1)^{2}}=b_{n}^{\prime} ; \\
c_{n}=\frac{1}{(n+1)^{2}}=c_{n}^{\prime}
\end{gathered}
$$


for all integers $n \geq 1$. Observe that if $T$ is Lipschitz, then

$$
\begin{aligned}
\alpha_{n} \beta_{n} \delta_{n} & =\sum \frac{1}{(n+1)} \delta_{n} \leq L^{2} \sum \frac{1}{(n+1)}\left(\beta_{n}+c_{n}^{\prime}\right)[\operatorname{diam}(K)]^{2} \\
& =L^{2} \sum \frac{1}{(n+1)}\left(\frac{1}{\sqrt{(n+1)}}+\frac{1}{(n+1)^{2}}\right)[\operatorname{diam}(K)]^{2}<\infty .
\end{aligned}
$$

3. Extensions of our theorems to set-valued maps are basically mere repetitions of our arguments (once a single-valued selection has been made) and are therefore omitted.

In connection with the iterative approximation of fixed points of pseudocontractions, the following question is still open.

Question. Does the Mann iteration process always converge for continuous pseudocontractions, or for even Lipschitz pseudocontractions?

Let $E$ be a Banach space and $K$ be a nonempty compact convex subset of $E$. Let $T: K \rightarrow K$ be a Lipschitz pseudocontractive map. Under this setting, even for $E=H$, a Hilbert space, the answer to the above question is not known. There is, however, an example of a discontinuous pseudocontractive map $T$ with a unique fixed point for which the Mann iteration process does not always converge to the fixed point of $T$.

Example (Hicks and Kubicek [14]). Let $H$ be the complex plane and $K:=\{z \in$ $H:|z| \leq 1\}$. Define $T: K \rightarrow K$ by

$$
T\left(r e^{i \theta}\right)= \begin{cases}2 r e^{i\left(\theta+\frac{\pi}{3}\right)}, & \text { for } 0 \leq r \leq \frac{1}{2}, \\ e^{i\left(\theta+\frac{2 \pi}{3}\right)}, & \text { for } \frac{1}{2}<r \leq 1 .\end{cases}
$$

Then, zero is the only fixed point of $T$. It is shown in [12] that $T$ is pseudocontractive and that with $c_{n}=\frac{1}{n+1}$, the sequence $\left\{z_{n}\right\}$ defined by $z_{n+1}=$ $\left(1-c_{n}\right) z_{n}+c_{n} T z_{n}, z_{0} \in K, n \geq 1$, does not converge to zero. Since the $T$ in this example is not continuous, the above question remains open.

\section{REFERENCES}

[1] F.E. Browder, Nonlinear operators and nonlinear equations of evolution in Banach spaces, Proc. of Symposia in Pure Math., Vol. XVIII, Part 2, 1976. MR 53:8982

[2] F.E. Browder and W.V. Petryshyn, Construction of fixed points of nonlinear mappings in Hilbert spaces, J. Math. Anal. Appl. 20 (1967), 197-228. MR 36:747

[3] C.E. Chidume, Iterative approximation of Lipschitz strictly pseudocontractive mappings, Proc. Amer. Math. Soc. 99 (2), (1987), 283-288. MR 87 m:47122

[4] C.E. Chidume, Approximation of fixed points of strongly pseudocontractive mappings, Proc. Amer. Math. Soc. 120 (2), (1994), 545-551. MR 94d:47056

[5] C.E. Chidume, Global iteration schemes for strongly pseudocontractive maps, to appear, Proc. Amer. Math. Soc. (1998). CMP 97:13

[6] C.E. Chidume, Iterative solution of nonlinear equations of strongly accretive type, Math. Nachr. 189 (1998), 49-60. CMP 98:07

[7] C.E. Chidume and Chika Moore, The solution by iteration of nonlinear equations in uniformly smooth Banach spaces, J. Math. Anal. Appl. 215 (1), (1997), 132-146. CMP 98:03

[8] C.E. Chidume and M.O. Osilike, Ishikawa iteration process for nonlinear Lipschitz strongly accretive mappings, J. Math. Anal. Appl. 192 (1995), 727-741. MR 96i:47099

[9] C.E. Chidume and M.O. Osilike, Nonlinear accretive and pseudocontractive operator equations in Banach spaces, Nonlinear Anal. TM 31 (7), (1998), 779-789. CMP 98:06

[10] M.G. Crandall and A. Pazy, On the range of accretive operators, Israel J. Math. 27 (1977), 235-246. MR 56:1142 
[11] L. Deng, On Chidume's open problems, J. Math. Anal. Appl. 174 (2), (1993), 441-449. MR 94b: 47073

[12] L. Deng, Iteration process for nonlinear Lipschitzian strongly accretive mappings in $L_{p}$ spaces, J. Math. Anal. Appl. 188 (1994), 128-140. MR 96f: 47124

[13] L. Deng and X.P. Ding, Iterative approximation of Lipschitz strictly pseudocontractive mappings in uniformly smooth Banach spaces, Nonlinear Anal. TMA 24 (7), (1995), 981-987. MR 96a:47096

[14] T.L. Hicks and J.R. Kubicek, On the Mann iteration process in Hilbert space, J. Math. Anal. Appl. 59 (1977), 498-504. MR 58:23802

[15] S. Ishikawa, Fixed point by a new iteration method, Proc. Amer. Math. Soc. 4 (1), (1974), 147-150. MR 49:1243

[16] L.S. Liu, Ishikawa and Mann iteration process with errors for nonlinear strongly accretive mappings in Banach spaces, J. Math. Anal. Appl. 194 (1995), 114-125. MR 97g:47069

[17] W.R. Mann, Mean value methods in iteration, Proc. Amer. Math. Soc. 4 (1953), 506-610. MR 14:988f

[18] Liu Qihou, On Naimpally and Singh's open questions, J. Math. Anal. Appl. 124 (1987), 157-164. MR 88j: 47078

[19] Liu Qihou, The convergence theorems of the sequence of Ishikawa iterates for hemicontractive mappings, J. Math. Anal. Appl. 148 (1990), 55-62. MR 92b:47094

[20] Liu Qihou, Convergence theorems of the sequence of iterates for asymptotically demicontractive and hemicontractive mappings, Nonlinear Anal. TMA 26 (11), (1996), 1835-1842. MR 97d:47069

[21] S. Reich, An iterative procedure for constructing zeros of accretive sets in Banach spaces, Nonlinear Anal. 2 (1978), 85-92. MR 81b:47065

[22] S. Reich, Constructive techniques for accretive and monotone operators in Applied Nonlinear Analysis, Academic Press, New York, (1979), 335-345. MR 80g:47059

[23] S. Reich, Constructing zeros of accretive operators, I II, Applicable Analysis 9 (1979), 159163. MR 82d:65052b

[24] S. Reich, Strong convergence theorems for resolvents of accretive operators in Banach spaces, J. Math. Anal. Appl. 75 (1980), 287-292. MR 82a:47050

[25] B.E. Rhoades, Comments on two fixed point iteration procedures, J. Math. Anal. Appl. 56 (1976), 741-750. MR 55:3885

[26] J. Schu, On a theorem of C.E. Chidume concerning the iterative approximation of fixed points, Math. Nachr. 153 (1991), 313-319. MR 93b:47123

[27] J. Schu, Iterative construction of fixed points of strictly pseudocontractive mappings, Applicable Analysis 40 (1991), 67-72. MR 92c: 47072

[28] J. Schu, Iterative construction of fixed points of asymptotically nonexpansive mappings, J. Math. Anal. Appl. 158 (1991), 407-413. MR 92d:47072

[29] Kok-Keong Tan and H.K. Xu, Approximating fixed points of nonexpansive mappings by the Ishikawa iteration process, J. Math. Anal. Appl. 178 (1993), 301-308. MR 94g:47076

[30] Yuguang $\mathrm{Xu}$, Ishikawa ana Mann iterative processes with errors for nonlinear strongly accretive operator equations, J. Math. Anal. Appl., (to appear).

[31] Z.B. Xu and G.F. Roach, A necessary and sufficient condition for convergence of steepest descent approximation to accretive operator equations, J. Math. Anal. Appl. 167 (1992), 340-354. MR 93e:47086

[32] X.L. Weng, Fixed point iteration for local strictly pseudocontractive mappings, Proc. Amer. Math. Soc. 113 (1991), 727-731. MR 92b:47099

The Abdus Salam International Centre for Theoretical Physics, Trieste, Italy

E-mail address, C. E. Chidume: chidume@ictp.trieste.it 